\title{
ORTHO-BASES AND MONOTONIC PROPERTIES
}

\author{
HEIKKI J. K. JUNNILA AND HANS-PETER A. KÜNZI
}

(Communicated by Franklin D. Tall)

\begin{abstract}
We study monotonic properties in the sense of Gartside and Moody. Among other things we show that each space with an ortho-base admits a monotonic quasi-uniformity and that the fine quasi-uniformity of a topological space which admits a monotonic quasi-uniformity need not be monotonic. Furthermore we give an example of a countable space that does not admit any monotonic quasi-uniformity. As a byproduct of our investigations we obtain a new characterization of the topological spaces having an ortho-base.
\end{abstract}

\section{INTRODUCTION}

All spaces considered are $T_{1}$-spaces. (Let us note however that in Proposition 4 we shall make use of topologies not satisfying the $T_{1}$-axiom.)

A diagonal (quasi-)uniformity $\mathscr{U}$ on a set $X$ is called monotonic $[4,1]$ if there exists an operator $M: \mathscr{U} \rightarrow \mathscr{U}$ such that $M(U) \subseteq M(V)$ whenever $U, V \in \mathscr{U}$ and $U \subseteq V$, and such that $M(U)^{2} \subseteq U$ whenever $U \in \mathscr{U}$. In [4] Gartside and Moody show that the topological spaces which admit a (separating) monotonic uniformity can be characterized as the proto-metrizable spaces, i.e., as the monotonically normal spaces having an ortho-base $[12,6]$. Furthermore they prove that the fine uniformity of each proto-metrizable space is monotonic.

It seems to be much harder to characterize those topological spaces that admit a monotonic quasi-uniformity (see [1]). Since clearly each quasi-uniformity with a base that is well ordered by reverse inclusion is monotonic, each quasimetrizable space belongs to this class. However, as is pointed out in [1], the class is much larger than one might first expect. In this note we show that each space with an ortho-base admits a monotonic quasi-uniformity. The fine quasiuniformity of a space with an ortho-base is monotonic if the space possesses an antisymmetric neighbornet. We prove on the other hand that the fine quasiuniformity of the real line is not monotonic. The same method of proof yields the corresponding result for the Sorgenfrey line and for $\omega_{1}$ (equipped with the

Received by the editors November 28, 1991 and, in revised form, April 5, 1992.

1991 Mathematics Subject Classification. Primary 54E15, 54D15, 54D20, 54E35.

Key words and phrases. Ortho-base, proto-metrizable, monotonically orthocompact, monotonically normal, monotonic quasi-uniformity.

This paper was written at the University of Helsinki while the second author was supported by the Swiss National Science Foundation under grants 8220-028387 and 20-27939.89. 
interval topology). Furthermore we construct a countable space that does not have any compatible monotonic quasi-uniformity.

In [5] Gartside and Moody show that proto-metrizable spaces can also be characterized by a certain monotonic covering property which they call monotone paracompactness: To each open cover $\mathscr{C}$ of a proto-metrizable space $X$ one can assign an open star-refinement $m(\mathscr{C})$ of $\mathscr{C}$ so that $m\left(\mathscr{C}_{1}\right)$ refines $m\left(\mathscr{C}_{2}\right)$ whenever $\mathscr{C}_{1}$ and $\mathscr{C}_{2}$ are open covers of $X$ and $\mathscr{C}_{1}$ refines $\mathscr{C}_{2}$. In this note we introduce a related monotonic covering property which we call monotone orthocompactness. We show that each space with an ortho-base satisfies our condition and that each monotonically normal monotonically orthocompact space is proto-metrizable. The question whether each monotonically orthocompact space has an ortho-base remains open.

Our results on ortho-bases suggest the concept of another interesting covering property stronger than orthocompactness which we call ortho-refinability. All spaces with an ortho-base and all submetacompact orthocompact spaces are ortho-refinable. Furthermore each ortho-refinable countably compact space is compact and each ortho-refinable monotonically normal space is paracompact. The authors do not know whether each ortho-refinable collectionwise normal space is paracompact.

For the basic facts about quasi-uniformities we refer the reader to [3]. Given collections $\mathscr{A}$ and $\mathscr{B}$ of subsets of a set $X$, we say that $\mathscr{A}$ is a partial refinement of $\mathscr{B}$ if each member of $\mathscr{A}$ is contained in some member of $\mathscr{B}$. A partial refinement $\mathscr{A}$ of $\mathscr{B}$ is said to be a refinement of $\mathscr{B}$ provided that $\bigcup \mathscr{A}=\bigcup \mathscr{B}$. A binary relation $S$ on a topological space $X$ is called a partial neighbornet on $X$ if for each $x \in X, S(x) \cup S^{-1}(x)=\varnothing$ or $S(x)$ is a neighborhood of $x$ in $X$. A partial neighbornet $N$ on a topological space $X$ is said to be unsymmetric [3, p. 4] if whenever $a, b \in X, a \in N(b)$, and $b \in N(a)$, then $N(b)=N(a)$.

We recall that a space $X$ is said to be monotonically normal [9] provided that there exists an operator $g(\cdot, \cdot)$ which assigns to each $x \in X$ and each open set $U$ containing $x$, an open set $g(x, U)$ also containing $x$ which satisfies: (1) $g(x, U) \subseteq g\left(x, U^{\prime}\right)$ whenever $x \in U \subseteq U^{\prime}$, and (2) $g(x, X \backslash\{y\}) \cap$ $g(y, X \backslash\{x\})=\varnothing$, if $x \neq y$.

A base $\mathscr{B}$ for a topological space $X$ is called an ortho-base [11] if whenever $\mathscr{B}^{\prime}$ is a subset of $\mathscr{B}, x \in \bigcap \mathscr{B}^{\prime}$, and $x \notin$ int $\bigcap \mathscr{B}^{\prime}$, then $\mathscr{B}^{\prime}$ is a local base at $x$. By Lemma 1.2 of [11] the collection of all open intersections of subcollections of an ortho-base is an ortho-base.

$\mathbf{N}$ will denote the set of positive integers. The diagonal of a topological space will be denoted by $\Delta$.

\section{MONOTONIC PROPERTIES RELATED TO ORTHOCOMPACTNESS}

We begin our discussion of monotonic properties related to orthocompactness with an unexpected characterization of lob spaces, which makes use of monotone families of neighbornets. Recall that a topological space is called a lob space [2] if each point has a local base which is linearly ordered by reverse inclusion.

Proposition 1. A topological space $X$ is a lob space if and only if there exists an operator $P: \Xi \rightarrow \mathscr{N}$ (where $\Xi$ is the set of all opencovers of $X$ and $\mathscr{N}$ is the 
set of all neighbornets of $X)$ such that $P\left(\mathscr{C}_{1}\right) \subseteq P\left(\mathscr{C}_{2}\right)$ whenever $\mathscr{C}_{1}, \mathscr{C}_{2} \in \Xi$ and $\mathscr{C}_{1}$ refines $\mathscr{C}_{2}$, and such that $\{P(\mathscr{C})(x): x \in X\}$ refines $\mathscr{C}$ whenever $\mathscr{C} \in \Xi$.

Proof. Let $X$ be a lob space. Then each point $x \in X$ has a local base $\mathscr{B}_{x}$ that is well ordered by reverse inclusion. Given a cover $\mathscr{C} \in \Xi$ choose for each $x \in X$ the first element $B_{x} \in \mathscr{B}_{x}$ that is contained in some member of $\mathscr{C}$ and set $P(\mathscr{C})=\bigcup_{x \in X}\left(\{x\} \times B_{x}\right)$. Clearly the operator $P: \Xi \rightarrow \mathscr{N}$ satisfies the stated conditions.

For the converse assume that $P: \Xi \rightarrow \mathcal{N}$ is an operator having the stated properties. Fix $x \in X$ and let $\mathscr{B}$ be any local base of open neighborhoods at $x$. For each nonempty subcollection $\mathscr{B}^{\prime}$ of $\mathscr{B}$ set $\mathscr{C}_{\mathscr{B}^{\prime}}=\{X \backslash\{x\}\} \cup \mathscr{B}^{\prime}$. Assume that for some ordinal $\beta$ we have defined elements $B_{\alpha} \in \mathscr{B}$ whenever $\alpha<\beta$. Then set $\mathscr{B}_{\beta}=\mathscr{B} \backslash\left\{B_{\alpha}: \alpha<\beta\right\}$ and $\mathscr{C}_{\beta}=\mathscr{C}_{\mathscr{B}_{\beta}}$. If $\mathscr{B}_{\beta} \neq \varnothing$, there is $B \in \mathscr{B}_{\beta}$ such that $P\left(\mathscr{C}_{\beta}\right)(x) \subseteq B$, since $\left\{P\left(\mathscr{C}_{\beta}\right)(y): y \in X\right\}$ refines the open cover $\mathscr{C}_{\beta}$ of $X$. Set $B_{\beta}=B$. The induction stops at some ordinal, say $\delta$, when $\mathscr{B}=\left\{B_{\alpha}: \alpha<\delta\right\}$. Clearly $\left\{P\left(\mathscr{C}_{\alpha}\right)(x): \alpha<\delta\right\}$ is a local base at $x$. Furthermore if $\alpha_{1}<\alpha_{2}<\delta$, then $\mathscr{C}_{\alpha_{2}} \subseteq \mathscr{C}_{\alpha_{1}}$ and thus $P\left(\mathscr{C}_{\alpha_{2}}\right)(x) \subseteq P\left(\mathscr{C}_{\alpha_{1}}\right)(x)$. Hence $X$ is a lob space.

Our next result will be crucial in the following.

Proposition 2. Let $X$ be a topological space with an ortho-base $\mathscr{B}$. We assume that $\mathscr{B}$ is closed under open intersections. Then there is an operator $T: \Gamma \rightarrow \mathscr{E}$ from the set $\Gamma$ of all open collections of $X$ to the set $\mathscr{E}$ of all transitive partial neighbornets on $X$ such that $T\left(\mathscr{C}_{1}\right) \subseteq T\left(\mathscr{C}_{2}\right)$ whenever $\mathscr{C}_{1}, \mathscr{C}_{2} \in \Gamma$ and $\mathscr{C}_{1}$ is a partial refinement of $\mathscr{C}_{2}$, and such that for each $\mathscr{C} \in \Gamma$ the collection $\{T(\mathscr{C})(x): x \in T(\mathscr{C})(X)\}$ is a subcollection of $\mathscr{B}$ and a refinement of $\mathscr{C}$.

Proof. Let $\mathscr{B}=\left\{B_{\alpha}: \alpha<\beta\right\}$ and $\mathscr{C} \in \Gamma$. Fix $x \in \cup \mathscr{C}$. Define $\alpha_{x}^{\mathscr{C}}$ as the minimal $\alpha<\beta$ such that $x \in B_{\alpha} \subseteq U$ for some $U \in \mathscr{C}$. Set $G(\mathscr{C})(x)=$ $\bigcap\left\{B_{\alpha}: x \in B_{\alpha}, \alpha \leq \alpha_{x}^{\mathscr{C}}\right\}$. Observe that $G(\mathscr{C})(x)$ is an open neighborhood of $x$ in $X$. Set $W(\mathscr{C})(x)=\bigcap\{B \in \mathscr{B}: x \in B, B \backslash G(\mathscr{C})(x) \neq \varnothing\}$ and $T(\mathscr{C})(x)=$ $W(\mathscr{C})(x) \cap G(\mathscr{C})(x)$. First let us verify that in fact $T(\mathscr{C})(x)=W(\mathscr{C})(x) \cap B_{\alpha_{x}^{8}}$. To this end consider an arbitrary $\alpha<\alpha_{x}^{\mathscr{C}}$ with $x \in B_{\alpha}$. If $B_{\alpha} \backslash G(\mathscr{C})(x)=\varnothing$, then $x \in B_{\alpha} \subseteq G(\mathscr{C})(x) \subseteq B_{\alpha_{x}^{\mathscr{E}}} \subseteq U$ for some $U \in \mathscr{C}$, contradicting the definition of $\alpha_{x}^{\mathscr{C}}$. Thus $B_{\alpha} \backslash G(\mathscr{C})(x) \neq \varnothing$ and $W(\mathscr{C})(x) \subseteq B_{\alpha}$. Therefore $T(\mathscr{C})(x) \subseteq W(\mathscr{C})(x) \cap B_{\alpha_{x}^{\mathscr{B}}} \subseteq W(\mathscr{C})(x) \cap G(\mathscr{C})(x) \subseteq T(\mathscr{C})(x)$ and we are finished.

Next we prove that the partial neighbornet $G(\mathscr{C})=\bigcup_{c \in \bigcup \mathscr{C}}(\{c\} \times G(\mathscr{C})(c))$ on $X$ is unsymmetric. Let $a, b \in \bigcup \mathscr{C}$ such that $a \in G(\mathscr{C})(b)$ and $b \in G(\mathscr{C})(a)$. We see that $\alpha_{a}^{\mathscr{C}}=\alpha_{b}^{\mathscr{C}}$, since for some $W, V \in \mathscr{C}$ we have $a \in B_{\alpha_{b}^{\mathscr{E}}} \subseteq V$ and $b \in B_{\alpha_{a}^{\mathscr{E}}} \subseteq W$. It follows that $G(\mathscr{C})(b)=G(\mathscr{C})(a)$, because $a \in G(\mathscr{C})(b)$ and $b \in G(\mathscr{C})(a)$.

Set $T(\mathscr{C})=\bigcup_{c \in \bigcup \mathscr{C}}(\{c\} \times T(\mathscr{C})(c))$. We are going to show that the partial neighbornet $T(\mathscr{C})$ on $X$ is transitive. Let $a, b \in \bigcup \mathscr{C}$ such that $a \in T(\mathscr{C})(b)$. Consider first the case that $G(\mathscr{C})(a)=G(\mathscr{C})(b)$. Since $a \in W(\mathscr{C})(b)$, it follows that $W(\mathscr{C})(a) \subseteq W(\mathscr{C})(b)$. Consequently $T(\mathscr{C})(a) \subseteq T(\mathscr{C})(b)$. Consider now the case that $G(\mathscr{C})(a) \neq G(\mathscr{C})(b)$. Since $a \in G(\mathscr{C})(b)$ and $G(\mathscr{C})$ is unsymmetric, we conclude that $b \notin G(\mathscr{C})(a)$. Therefore $T(\mathscr{C})(a) \subseteq W(\mathscr{C})(a) \subseteq$ 
$W(\mathscr{C})(b) \cap B_{\alpha_{b}^{\mathscr{E}}}=T(\mathscr{C})(b)$, since $a \in W(\mathscr{C})(b) \cap B_{\alpha_{b}^{\mathscr{E}}}$. Thus $T(\mathscr{C})$ is transitive. Note also that $\{T(\mathscr{C})(x): x \in T(\mathscr{C})(X)\}$ is a subcollection of $\mathscr{B}$ and a refinement of $\mathscr{C}$. It remains to show that the operator $T$ is monotone.

Let $\mathscr{C}, \mathscr{D} \in \Gamma$ such that $\mathscr{C}$ is a partial refinement of $\mathscr{D}$. Fix $y \in \cup \mathscr{C}$. There exist $C \in \mathscr{C}$ and $D \in \mathscr{D}$ such that $y \in B_{\alpha_{y}^{\mathscr{E}}} \subseteq C \subseteq D$. Consequently $a_{y}^{\mathscr{D}} \leq \alpha_{y}^{\mathscr{C}}, G(\mathscr{C})(y) \subseteq G(\mathscr{D})(y), W(\mathscr{C})(y) \subseteq W(\mathscr{D})(y)$, and $T(\mathscr{C})(y) \subseteq$ $T(\mathscr{D})(y)$. We have proved that $T(\mathscr{C}) \subseteq T(\mathscr{D})$.

Definition 1. A topological space $X$ is called monotonically orthocompact provided that there is an operator $T: \Xi \rightarrow \mathscr{S}$ from the set $\Xi$ of all open covers of $X$ to the set $\mathscr{S}$ of all transitive neighbornets of $X$ such that $T(\mathscr{C}) \subseteq T(\mathscr{D})$ whenever $\mathscr{C}, \mathscr{D} \in \Xi$ and $\mathscr{C}$ is a refinement of $\mathscr{D}$, and such that $\{T(\mathscr{C})(x)$ : $x \in X\}$ refines $\mathscr{C}$ whenever $C \in \Xi$.

Corollary 1. Each topological space $X$ with an ortho-base is hereditarily monotonically orthocompact.

Corollary 2. Let $X$ be a topological space with an ortho-base. Then there is an operator $L: \mathscr{R} \rightarrow \mathscr{S}$ from the set $\mathscr{R}$ of all unsymmetric neighbornets of $X$ to the set $\mathscr{S}$ of all transitive neighbornets of $X$ such that $L(U) \subseteq L(V)$ whenever $U, V \in \mathscr{R}$ and $U \subseteq V$, and such that $L(U) \subseteq U$ whenever $\bar{U} \in \mathscr{R}$.

Proof. Let $\mathscr{B}=\left\{B_{\alpha}: \alpha<\beta\right\}$ be an ortho-base for $X$ that is closed under open intersections and let $U \in \mathscr{R}$. For any $x \in X$ let $\alpha_{x}^{U}$ be the minimal $\alpha<\beta$ such that $x \in B_{\alpha} \subseteq U(x)$. Define now $L(U)$ like $T(\mathscr{C})$ in the proof of the preceding proposition for an open cover $\mathscr{C}$ of $X$. One readily checks that the operator $L$ has all the stated properties.

Corollary 3. Each space $X$ with an ortho-base and an antisymmetric neighbornet $S$ (i.e., $S \cap S^{-1}=\Delta$ ) has a fine quasi-uniformity which is monotonic. (The fine quasi-uniformity of $X$ is equal to the set of all neighbornets of $X$.)

Proof. Let $V$ be an arbitrary neighbornet of $X$. Observe that $P_{V}=V \cap S$ is an unsymmetric neighbornet. Set $M(V)=L\left(P_{V}\right)$, where $L$ is an operator as defined in Corollary 2. Clearly the operator $M$ witnesses that the fine quasiuniformity of $X$ is monotonic.

Let us note that, e.g., the Michael line [11, Example 5.1] satisfies the hypothesis of Corollary 3.

Proposition 3. A space $X$ is proto-metrizable if and only if it is monotonically orthocompact and monotonically normal.

Proof. If $X$ is proto-metrizable, then $X$ has an ortho-base and is monotonically normal . Hence one part of the assertion follows from Corollary 1. It remains to show that $X$ is proto-metrizable provided that $X$ is monotonically orthocompact and monotonically normal. By the results of [4] it suffices to prove that to any neighborhood $U$ of the diagonal $\Delta$ of $X$ one can assign a neighborhood $M(U)$ of $\Delta$ such that $M\left(U_{1}\right) \subseteq M\left(U_{2}\right)$ whenever $U_{1}$ and $U_{2}$ are neighborhoods of $\Delta$ with $U_{1} \subseteq U_{2}$, and such that $M(U)^{2} \subseteq U$ for each $U$. Therefore, let $U$ be an arbitrary neighborhood of $\Delta$. Set $\mathscr{C}_{U}=\{G: G \times G \subseteq U$ and $G$ is open in $X\}$. Since $X$ is monotonically orthocompact, we can assign to $U$ a transitive neighbornet $T\left(\mathscr{C}_{U}\right)$ of $X$ such that $T\left(\mathscr{C}_{U_{1}}\right) \subseteq T\left(\mathscr{C}_{U_{2}}\right)$ whenever $U_{1}, U_{2}$ are neighborhoods of $\Delta$ with $U_{1} \subseteq U_{2}$, and such that for each 
$U,\left\{T\left(\mathscr{C}_{U}\right)(x): x \in X\right\}$ is a refinement of the open cover $\mathscr{C}_{U}$ of $X$. Set

$$
M(U)=\bigcup_{x \in X}\left[g\left(x, T\left(\mathscr{C}_{U}\right)(x)\right) \times g\left(x, T\left(\mathscr{C}_{U}\right)(x)\right)\right],
$$

where $g(\cdot, \cdot)$ is an operator that witnesses monotone normality of $X$ (see Introduction). One readily checks that the neighborhoods $M(U)$ of $\Delta$ satisfy all the necessary conditions. Hence $X$ is proto-metrizable.

In the proof of our next proposition we shall need the following auxiliary result. It makes use of a generalization of a concept due to Kofner (see [10, Definition 6]).

Definition 2. A pair-base $\mathscr{P}$ of a topological space $X$ is called a preortho-pairbase provided that for each subcollection $\mathscr{P}_{0}$ of $\mathscr{P}$ and each $x \in \bigcap\left\{G^{\prime}\right.$ : $\left.\left(G^{\prime}, G^{\prime \prime}\right) \in \mathscr{P}_{0}\right\}$ such that $x \notin \operatorname{int} \bigcap\left\{G^{\prime \prime}:\left(G^{\prime}, G^{\prime \prime}\right) \in \mathscr{P}_{0}\right\}$, the collection $\left\{G^{\prime}:\left(G^{\prime}, G^{\prime \prime}\right) \in \mathscr{P}_{0}\right\}$ is a local base at $x$.

It is straightforward to verify that each space with a preortho-pair-base is preorthocompact (see [3] for the definition of this concept).

Lemma 1. A space $X$ is proto-metrizable if and only if it is monotonically normal and has a preortho-pair-base.

Proof. By [7, Theorem 2.4] a space is proto-metrizable if and only if it has a rank-1 pair-base. (A pair-base $\mathscr{B}$ is called a rank-1 pair-base provided that $B_{1}^{\prime} \subseteq B_{2}^{\prime \prime}$ or $B_{2}^{\prime} \subseteq B_{1}^{\prime \prime}$ whenever $\left(B_{1}^{\prime}, B_{1}^{\prime \prime}\right),\left(B_{2}^{\prime}, B_{2}^{\prime \prime}\right) \in \mathscr{B}$ and $B_{1}^{\prime} \cap B_{2}^{\prime} \neq \varnothing$.) We first show that each rank-1 pair-base $\mathscr{B}$ of a topological space is a preorthopair-base. Let $\mathscr{B}_{0} \subseteq \mathscr{B}, x \in \bigcap\left\{B^{\prime}:\left(B^{\prime}, B^{\prime \prime}\right) \in \mathscr{B}_{0}\right\}$, and suppose that $\left\{B^{\prime}:\left(B^{\prime}, B^{\prime \prime}\right) \in \mathscr{B}_{0}\right\}$ is not a local base at $x$. Hence there is a neighborhood $U$ of $x$ such that $B^{\prime} \nsubseteq U$ whenever $\left(B^{\prime}, B^{\prime \prime}\right) \in \mathscr{B} 0$. Choose $\left(Q^{\prime}, Q^{\prime \prime}\right) \in \mathscr{B}$ such that $x \in Q^{\prime} \subseteq Q^{\prime \prime} \subseteq U$. Then for each $\left(B^{\prime}, B^{\prime \prime}\right) \in \mathscr{B}_{0}$ we have $B^{\prime} \cap Q^{\prime} \neq \varnothing$, $B^{\prime} \nsubseteq Q^{\prime \prime}$, and thus $Q^{\prime} \subseteq B^{\prime \prime}$. Therefore $x \in$ int $\bigcap\left\{B^{\prime \prime}:\left(B^{\prime}, B^{\prime \prime}\right) \in \mathscr{B}_{0}\right\}$. We have shown that $\mathscr{B}$ is a preortho-pair-base. Hence each proto-metrizable space is monotonically normal and has a preortho-pair-base.

For the converse assume that $X$ has a preortho-pair-base $\mathscr{B}$ and is monotonically normal. We construct a rank-1 pair-base. To this end for each open set $U$ of $X$ and any point $x \in U$ set $h(x, U)=\operatorname{int} \cap\left\{B^{\prime \prime}: x \in B^{\prime},\left(B^{\prime}, B^{\prime \prime}\right) \in \mathscr{B}\right.$ and $\left.B^{\prime} \nsubseteq U\right\}$ and $q(x, U)=g(x, U) \cap h(x, U)$, where $g(\cdot, \cdot)$ is an operator witnessing monotone normality of $X$. Imitating the proof of Theorem 5 of [6] we check that $\mathscr{Q}=\left\{\left(q\left(x, B^{\prime}\right), B^{\prime \prime}\right): x \in B^{\prime},\left(B^{\prime}, B^{\prime \prime}\right) \in \mathscr{B}\right\}$ is a rank-1 pairbase: Clearly $\mathscr{Q}$ is a pair-base. Suppose that $q\left(x, B_{1}^{\prime}\right) \cap q\left(y, B_{2}^{\prime}\right) \neq \varnothing$, where $\left(B_{1}^{\prime}, B_{1}^{\prime \prime}\right),\left(B_{2}^{\prime}, B_{2}^{\prime \prime}\right) \in \mathscr{B}$ and $x \in B_{1}^{\prime}, y \in B_{2}^{\prime}$. Without loss of generality we may assume that $x \in B_{2}^{\prime}$. If $B_{2}^{\prime} \subseteq B_{1}^{\prime \prime}$, then $q\left(y, B_{2}^{\prime}\right) \subseteq g\left(y, B_{2}^{\prime}\right) \subseteq B_{2}^{\prime} \subseteq B_{1}^{\prime \prime}$. On the other hand, if $B_{2}^{\prime} \nsubseteq B_{1}^{\prime \prime}$, then $q\left(x, B_{1}^{\prime}\right) \subseteq h\left(x, B_{1}^{\prime}\right) \subseteq h\left(x, B_{1}^{\prime \prime}\right) \subseteq B_{2}^{\prime \prime}$. Hence $\mathscr{Q}$ is a rank-1 pair-base and $X$ is proto-metrizable.

Proposition 4. Let $(X, \leq)$ be a linearly ordered set, and let $\mathscr{P}$ be the Pervin quasi-uniformity of the upper topology $\mathscr{O}$ generated by the subbase \{]$a, \rightarrow[:$ $a \in X\}$ on $X$. Denote by $\mathscr{P} S$ the coarsest uniformity on $X$ finer than $\mathscr{P}$ and by $\mathscr{N}$ the set of all $\mathscr{T}(\mathscr{P} S)$-neighbornets of $X$. Then there exists an operator $M: \mathscr{P} \rightarrow \mathcal{N}$ satisfying both (1) $M(U) \subseteq M(V)$ whenever $U, V \in \mathscr{P}$ and 
$U \subseteq V$, and (2) $M(U)^{2} \subseteq U$ whenever $U \in \mathscr{P}$, if and only if the topological space $(X, \mathscr{T}(\mathscr{P} S))$ has an ortho-base.

Proof. Observe first that \{]$\leftarrow, a]: a \in X\} \cap \mathscr{O}$ is a subbase for the topology $\mathscr{T}(\mathscr{P} S)$. Assume that there exists an operator $M: \mathscr{P} \rightarrow \mathcal{N}$ with the stated properties. Set $\left.\left.S_{a}=(] \leftarrow, a\right] \times X\right) \cup(X \times] a, \rightarrow[)$ whenever $a \in X$. Let $x, y \in X$ with $y<x$. Set $G_{x y}=(X \times X) \backslash\{(x, y)\}$. Note that $G_{x y} \in \mathscr{P}$, since $S_{y} \in \mathscr{P}$ and $S_{y} \subseteq G_{x y}$. There exists an interval $I_{x y}$ with (missing) left endpoint $\psi(x, y)$ such that $x \in I_{x y} \subseteq M\left(G_{x y}\right)(x)$. Observe that $y \leq \psi(x, y)<x$, since $M\left(G_{x y}\right) \subseteq G_{x y}$.

Fix $a \in X$ where $a$ is not minimal in $X$. We claim that there exists $\alpha_{a} \in X$ such that $\alpha_{a}<a$ and $\psi(] a, \rightarrow[\times] a_{a}, a[) \subseteq[a, \rightarrow[$. Assume the contrary. Since $M\left(S_{a}\right)(a)$ is a $\mathscr{T}(\mathscr{P} S)$-neighborhood of $a$, by our assumption there exist $x, y \in X$ such that $y<a<x, \psi(x, y)<a$, and $y \in M\left(S_{a}\right)(a)$. Because $M$ is monotone and $S_{a} \subseteq G_{x y}$, it follows that $y \in M\left(S_{a}\right)(a) \subseteq M\left(G_{x y}\right)(a)$. Since $a \in I_{x y} \subseteq M\left(G_{x y}\right)(x)$, we deduce that $y \in G_{x y}(x)$-a contradiction. Consequently our assertion is verified.

Set $\mathscr{B}=\{(] \psi(x, y), x],] y, x]): x, y \in X$ and $y<x\}$. (Add the pair $(\{m\},\{m\})$ in the case that $X$ has a minimal element $m$.) Clearly $\mathscr{B}$ is a pair-base for $(X, \mathscr{T}(\mathscr{P} S))$. Let us show that $\mathscr{B}$ is a preortho-pair-base for $\left(X, \mathscr{T}\left(\mathscr{P}^{S}\right)\right)$. Suppose that $\left.\left.z \in \bigcap\{] \psi(x, y), x\right]:(x, y) \in M\right\}$ for some subset $M$ of $\{(x, y) \in X \times X: y<x\}$ and that there does not exist any $\beta_{z} \in X$ such that for all $y \in\{u:(x, u) \in M$ for some $x \in X\}$ we have $y<\beta_{z}<z$. It follows from the property of $\psi$ established above that $(x, y) \in M$ and $\alpha_{z}<y$ imply that $x=z$, because $y \leq \psi(x, y) \leq z \leq x$. We conclude that $\{\mathrm{l} y, x]:(x, y) \in M\}$ is a local $\mathscr{T}(\mathscr{P} S)$-base at $z$. Therefore $\mathscr{B}$ is a preorthopair-base for $(X, \mathscr{T}(\mathscr{P} S))$. Since $(X, \mathscr{T}(\mathscr{P} S))$ is a generalized ordered space and hence monotonically normal [9, Corollary 5.6], we see by Lemma 1 that $(X, \mathscr{T}(\mathscr{P} S))$ is proto-metrizable and thus has an ortho-base.

For the converse suppose that $(X, \mathscr{T}(\mathscr{P} S))$ has an ortho-base. Clearly the space $(X, \mathscr{T}(\mathscr{P} S))$ has an antisymmetric neighbornet. Hence by Corollary 3 the fine quasi-uniformity $\mathscr{N}$ of $(X, \mathscr{T}(\mathscr{P} S))$ is monotonic. Since $P \subseteq \mathscr{N}$, we conclude that there exists an operator $M: \mathscr{P} \rightarrow \mathscr{N}$ with the stated properties.

Corollary 4. The Pervin quasi-proximity class of the real line, the Sorgenfrey line, and the space $\omega_{1}$ (equipped with the interval topology) do not contain any monotonic quasi-uniformities.

Proof. Let $\mathscr{P}$ be the Pervin quasi-uniformity of the upper topology on the corresponding linearly ordered set $X$, and let $\mathscr{N}$ be the set of all $\mathscr{T}(\mathscr{P} S)$ neighbornets of $X$. If $\mathscr{U}$ is a monotonic quasi-uniformity on $X$ with a monotone operator $N: \mathscr{U} \rightarrow \mathscr{U}$ and $\mathscr{P} \subseteq \mathscr{U} \subseteq \mathscr{N}$, then the restriction of $N$ to $\mathscr{P}$ is an operator $M$ of the kind described in Proposition 4. However such an $M$ cannot exist by Proposition 4, since neither the Sorgenfrey line [11, Example 5.2] nor the space $\omega_{1}[6$, Theorem 3] has an ortho-base.

Let us note that by Proposition 3, the Sorgenfrey line is not monotonically orthocompact, although it admits a monotonic quasi-uniformity, since it is quasimetrizable. We do not know whether each monotonically orthocompact space admits a monotonic quasi-uniformity nor do we know whether each monotonically orthocompact space has an ortho-base. With the help of the next result, 
however, we show below that each topological space with an ortho-base admits a monotonic quasi-uniformity. The reader should compare our next proposition with similar results on proto-uniformities mentioned in [12]. (In the following we set $\mathscr{T}_{\alpha}=\left\{T_{\alpha}(x): x \in T_{\alpha}(X)\right\}$ for a given partial neighbornet $T_{\alpha}$ on a space $X$.)

Proposition 5. A topological space $X$ has an ortho-base if and only if there exists a decreasing chain $\left(T_{\alpha}\right)_{\alpha<\delta}$ of transitive partial neighbornets on $X$ such that for each $x \in X,\left\{\operatorname{st}\left(x, \mathscr{T}_{\alpha}\right): x \in \bigcup \mathscr{T}_{\alpha}, \alpha<\delta\right\}$ is a local base at $x$.

Proof. Assume that $X$ has a decreasing chain $\left(T_{\alpha}\right)_{\alpha<\delta}$ of transitive partial neighbornets having the stated property. Set $\mathscr{B}=\left\{T_{\alpha}(x): x \in T_{\alpha}(X), \alpha<\delta\right\}$. We show that $\beta$ is an ortho-base for $X$. Obviously $\mathscr{B}$ is a base for $X$. Assume that $\mathscr{B}^{\prime} \subseteq \mathscr{B}, x \in \bigcap \mathscr{B}^{\prime}$, but $x \notin$ int $\bigcap \mathscr{B}^{\prime}$. Let $\beta$ be the minimal $\gamma \leq \delta$ such that $\mathscr{B}^{\prime} \subseteq \bigcup_{\alpha<\gamma} \mathscr{T}_{\alpha}$. If $x \in \bigcup \mathscr{T}_{\beta}$, then $T_{\beta}(x) \subseteq \bigcap \mathscr{B}^{\prime}-$ a contradiction. Consequently $x \notin \bigcup \mathscr{T}_{\beta}$. Thus $\mathscr{B}^{\prime}$ clearly is a local base at $x$. We have shown that $\mathscr{B}$ is an ortho-base for $X$.

For the converse suppose that $X$ has an ortho-base $\mathscr{B}$. We assume that $\mathscr{B}$ is closed under open intersections. Then there exists an operator $T: \Gamma \rightarrow \mathscr{E}$ as described in Proposition 2. Let $\mathscr{C}_{0}$ be the topology of $X$. Assume that $\mathscr{C}_{\alpha} \in \Gamma$ is defined for some ordinal $\alpha$. If possible, choose $G_{0} \in \mathscr{C}_{\alpha}$ such that $G_{0}$ is not a singleton and set $\mathscr{C}_{\alpha+1}=\mathscr{C}_{\alpha} \backslash\left\{G \in \mathscr{C}_{\alpha}: G_{0} \subseteq G\right\}$. For a limit ordinal $\gamma$ set $\mathscr{C}_{\gamma}=\bigcap_{\alpha<\gamma} \mathscr{C}_{\alpha}$. Obviously this inductive construction stops at some ordinal, say $\varepsilon$, when $\mathscr{C}_{\varepsilon}$ is the collection of all isolated singletons of $X$. In light of Proposition 2 it remains only to show that the decreasing chain $\left(T\left(\mathscr{C}_{\alpha}\right)\right)_{\alpha \leq \varepsilon}$ of transitive partial neighbornets on $X$ satisfies the stated local base condition. Let $x \in X$. We can assume that $x$ is not isolated in $X$, because for isolated points the condition trivially holds, since $T\left(\mathscr{C}_{\varepsilon}\right)(x)=\{x\}$ for any $x \in \bigcup \mathscr{C}_{\varepsilon}$. Let $p$ be the minimal $\alpha \leq \varepsilon$ such that $x \notin T\left(\mathscr{C}_{\alpha}\right)(X)$. Note that $\rho$ is a limit ordinal, since $T\left(\mathscr{C}_{\alpha}\right)(X)=T\left(\mathscr{C}_{\alpha+1}\right)(X)$ whenever $\alpha<\varepsilon$. Assume that there exists a neighborhood $U$ of $x$ such that for each $\gamma<\rho$ there exists $y_{\gamma} \in X$ such that $x \in T\left(\mathscr{C}_{\gamma}\right)\left(y_{\gamma}\right)$ and $T\left(\mathscr{C}_{\gamma}\right)\left(y_{\gamma}\right) \nsubseteq U$. Since each $T\left(\mathscr{C}_{\gamma}\right)\left(y_{\gamma}\right) \in \mathscr{B}$, we conclude that there exists an open set $G$ in $X$ such that $x \in G \subseteq \bigcap_{\gamma<\rho} T\left(\mathscr{C}_{\gamma}\right)\left(y_{\gamma}\right)$. Observe that for each $\gamma<\rho$, there exists $C_{\gamma} \in \mathscr{C}_{\gamma}$ such that $T\left(\mathscr{C}_{\gamma}\right)\left(y_{\gamma}\right) \subseteq C_{\gamma}$. Furthermore there exists $\alpha_{0}<\varepsilon$ such that $G \in \mathscr{C}_{\alpha_{0}}$, but $G \notin \mathscr{C}_{\alpha_{0}+1}$. Because each $C_{\gamma}$ contains $G$ as a subset, $C_{\gamma} \notin \mathscr{C}_{\alpha_{0}+1}$ and thus $\gamma \leq \alpha_{0}$ whenever $\gamma<\rho$. Since $\rho$ is a limit ordinal, we deduce that $\rho \leq \alpha_{0}$. Thus $x \in G \in \mathscr{C}_{\alpha_{0}} \subseteq \mathscr{C}_{\rho}$ and $x \in \bigcup \mathscr{C}_{\rho}=T\left(\mathscr{C}_{\rho}\right)(X)$-a contradiction. We conclude that $\left\{\right.$ st $\left.\left(x, \mathscr{T}_{\alpha}\right): x \in \bigcup \mathscr{T}_{\alpha}, \alpha \leq \varepsilon\right\}$ is a local base at $x$.

Proposition 6. Each topological space $X$ with an ortho-base admits a monotonic quasi-uniformity.

Proof. By Proposition 5 there exists a decreasing chain $\left(T_{\alpha}\right)_{\alpha<\delta}$ of transitive partial neighbornets on $X$ such that for each $x \in X,\left\{\operatorname{st}\left(x, \mathscr{T}_{\alpha}\right): x \in \bigcup \mathscr{T}_{\alpha}\right.$, $\alpha<\delta\}$ is a local base at $x$. For each map $a \in \delta^{X}$ such that $x \in T_{a(x)}(X)$ whenever $x \in X$, set $U_{a}=\bigcup_{x \in X}\left(T_{a(x)}^{-1}(x) \times T_{a(x)}(x)\right)$ and let $\mathscr{U}$ be the filter on $X \times X$ generated by the filterbase consisting of all such relations $U_{a}$. Observe that each $U_{a}$ is a transitive neighbornet of $X$. Let $U \in \mathscr{U}$ and for each $x \in X$ let $e(x)$ be the minimal $\beta<\delta$ such that $T_{\beta}^{-1}(x) \times T_{\beta}(x) \subseteq U$. Set $M(U)=U_{e}$. Then $U_{e} \in \mathscr{U}$ and $M: \mathscr{U} \rightarrow \mathscr{U}$ is monotone. We conclude that 
$\mathscr{U}$ is a monotonic quasi-uniformity on $X$. Let $y \in X$ and let $\alpha \in \delta$ such that $y \in \mathscr{T}_{\alpha}(X)$. By the local base property of the chain $\left(T_{\alpha}\right)_{\alpha<\delta}$, for each $x \in X \backslash\{y\}$ there exists $c(x)<\delta$ such that $x \in T_{c(x)}(X)$ and $x \notin T_{c(x)}(y)$. Set $c(y)=\alpha$. Then $U_{c} \in \mathscr{U}$ and $U_{c}(y)=T_{\alpha}(y)$. We conclude that the quasi-uniformity $\mathscr{U}$ is compatible with the topology of $X$.

Let us note that the constructed quasi-uniformity $\mathscr{U}$ contains all neighborhoods of the diagonal of $X$.

While Proposition 4 provides us with simple examples of spaces whose fine quasi-uniformity is not monotonic, our next proposition and (the related) Corollary 3 proved above show that nevertheless the fine quasi-uniformity of many (countable) spaces is monotonic.

Proposition 7. Each space $X$ with a closure-preserving cover $\mathscr{F}$ by finite sets and with the property that each point $x \in X$ has a local base $\mathscr{B}_{x}$ of open neighborhoods such that $\mathscr{B}_{x} \mid(X \backslash\{x\})$ is interior-preserving, has a fine quasiuniformity which is monotonic.

Proof. Let $x \in X$. Set $T(x)=X \backslash \bigcup\{F \in \mathscr{F}: x \in(X \backslash F)\}$. Observe that $T^{-1}(x)=\bigcap\{F: x \in F \in \mathscr{F}\}$, hence $T^{-1}(x)$ is finite. Consider an arbitrary neighbornet $U$ of $X$. For each $x \in X$ set $S(x)=U(x) \cap T(x) \cap(\bigcap\{B \in$ $\left.\left.\bigcup_{y \in T^{-1}(x), y \neq x} \mathscr{B}_{y}: x \in B\right\}\right)$ and $M(U)(x)=\bigcup\left\{B \in \mathscr{B}_{x}: B \subseteq S(x)\right\}$. Let $M(U)=\bigcup_{x \in X}(\{x\} \times M(U)(x))$. Consider $x, y \in X$ with $x \neq y$ and $y \in$ $M(U)(x)$. Since $x \in T^{-1}(y)$, it follows that $M(U)(y) \subseteq S(y) \subseteq M(U)(x)$. Therefore $M(U) \subseteq U$ is a transitive neighbornet of $X$. Note that whenever $V$ and $W$ are neighbornets of $X$ with $V \subseteq W$, then $M(V) \subseteq M(W)$. We conclude that the fine quasi-uniformity of $X$ is monotonic.

The following example shows that even for countable spaces we cannot drop the condition on local bases formulated in Proposition 7.

Example 1. We construct a countable space $X$ that does not admit a monotonic quasi-uniformity. Let $X=\{\infty\} \cup \mathbf{N} \cup \mathbf{N}^{2}$. The points of $\mathbf{N}^{2}$ are isolated. For each $n \in \mathbf{N}$ let $\left\{O_{k}(n): k \in \mathscr{N}\right\}$ be a local base at $n$, where $O_{k}(n)=\{n\} \cup\{(n, l): l \in \mathbf{N}$ and $l \geq k\}$ whenever $k \in \mathbf{N}$. A typical neighborhood of $\infty$ is $\{\infty\} \cup \bigcup\left\{O_{k_{n}}(n): n \in \mathbf{N} \backslash A\right\}$, where $\left(k_{n}\right)_{n \in \mathbf{N}}$ is any sequence in $\mathbf{N}$ and $A$ is any finite subset of $\mathbf{N}$. For later use, for each $n, k \in \mathbf{N}$ set $G_{n k}=X \backslash\{(n, l): l \in \mathbf{N}$ and $l \leq k\}$ and $G_{n}=\bigcap_{k \in \mathbf{N}} G_{n k}$. Of course, each set $G_{n}$ is a neighborhood of $\infty$. Suppose that $\mathscr{U}$ is a compatible monotonic quasiuniformity on $X$ with a monotone operator $M: \mathscr{U} \rightarrow \mathscr{U}$. Note that for any neighborhood $G$ of $\infty$ the relation $U_{G}=[(X \backslash\{\infty\}) \times X] \cup[\{\infty\} \times G]$ belongs to $\mathscr{U}$, since $\mathscr{U}$ is compatible. For our convenience let us set $G^{\prime}=M\left(U_{G}\right)(\infty)$. First we prove that for any $n \in \mathbf{N}$ there is $k \in \mathbf{N}$ such that $n \notin G_{n k}^{\prime}$. Assume the contrary. Then there is $m \in \mathbf{N}$ such that $m \in \bigcap_{k \in \mathbf{N}} G_{m k}^{\prime}$. Consequently for each $k \in \mathbf{N}$, we have $M\left(U_{G_{m k}}\right)(m) \subseteq U_{G_{m k}}(\infty)=G_{m k}$, because $\left(M\left(U_{G_{m k}}\right)\right)^{2} \subseteq U_{G_{m k}}$. Since $G_{m}=\bigcap_{k \in \mathbf{N}} G_{m k}$, clearly $U_{G_{m}}=\bigcap_{k \in \mathbf{N}} U_{G_{m k}}$. Hence $M\left(U_{G_{m}}\right) \subseteq \bigcap_{k \in \mathbf{N}} M\left(U_{G_{m k}}\right)$, because $M$ is monotone. Thus $M\left(U_{G_{m}}\right)(m) \subseteq$ $\bigcap_{k \in \mathbf{N}} M\left(U_{G_{m k}}\right)(m) \subseteq \bigcap_{k \in \mathbf{N}} G_{m k}$. But this is impossible, because $\bigcap_{k \in \mathbf{N}} G_{m k}$ is not a neighborhood of $m$. Therefore we have shown that for each $n \in \mathbf{N}$ there is $k_{n} \in \mathbf{N}$ such that $n \notin G_{n k_{n}}^{\prime}$. Observe that $G_{*}=\bigcap_{n \in \mathbf{N}} G_{n k_{n}}$ is a neighborhood of $\infty$. Consequently $G_{*}^{\prime}$ is defined, and it follows by an argument 
similar to the one given above that $G_{*}^{\prime} \subseteq \bigcap_{n \in \mathbf{N}} G_{n k_{n}}^{\prime}$. However $G_{*}^{\prime}$ is not a neighborhood of $\infty$, because $G_{*}^{\prime} \cap \mathbf{N}=\varnothing$. We have reached a contradiction and conclude that $X$ does not admit any monotonic quasi-uniformity.

\section{ORTHO-REFINABILITY}

In light of Proposition 5 it seems worthwhile to study the following covering property of topological spaces more thoroughly.

Definition 3. A space $X$ is called ortho-refinable provided that for each open cover $\mathscr{C}$ of $X$ there are an ordinal $\delta$ and a decreasing chain $\left(T_{\alpha}\right)_{\alpha<\delta}$ of transitive partial neighbornets on $X$ so that for each $x \in X$ there exists $\alpha<\delta$ such that $x \in \operatorname{st}\left(x, \mathscr{T}_{\alpha}\right) \subseteq C$ for some $C \in \mathscr{C}$. (Here, as usual, $\mathscr{T}_{\alpha}=\left\{T_{\alpha}(x)\right.$ : $\left.x \in T_{\alpha}(X)\right\}$.)

By Proposition 5 each space with an ortho-base is (hereditarily) orthorefinable. We conclude this paper with some further results on ortho-refinability.

Proposition 8. Each ortho-refinable space $X$ is orthocompact.

Proof. Given an open cover $\mathscr{C}$ of $X$ and a decreasing chain $\left(T_{\alpha}\right)_{\alpha<\delta}$ of transitive partial neighbornets on $X$ witnessing ortho-refinability of $X$ with respect to $\mathscr{C}$, we set $\alpha_{x}=\min \left\{\alpha<\delta: \operatorname{st}\left(x, \mathscr{T}_{\alpha}\right) \subseteq C\right.$ for some $\left.C \in \mathscr{C}\right\}$ and $\mathscr{D}=\left\{T_{a_{x}}(x): x \in X\right\}$. It remains to show that the open refinement $\mathscr{D}$ of $\mathscr{C}$ is interior-preserving. To this end let $x, y \in X$ such that $y \in T_{\alpha_{x}}(x)$. If $\alpha_{y}<\alpha_{x}$, then $\operatorname{st}\left(x, \mathscr{T}_{\alpha_{y}}\right) \subseteq \operatorname{st}\left(y, \mathscr{T}_{\alpha_{y}}\right) \subseteq C-$ a contradiction. Hence $\alpha_{y} \geq \alpha_{x}$ and $T_{\alpha_{y}}(y) \subseteq T_{\alpha_{x}}(y) \subseteq T_{\alpha_{x}}(x)$.

Proposition 9. Each orthocompact submetacompact (in particular, each metacompact) space $X$ is ortho-refinable.

Proof. Let $\mathscr{C}$ be an open cover of $X$, and let $\left(\mathscr{G}_{n}\right)_{n \in \mathbf{N}}$ be a $\theta$-sequence of open refinements of $\mathscr{C}$. Without loss of generality we assume that each $\mathscr{G}_{n}$ is interior-preserving. For each $n, k \in \mathbf{N}$ set $\mathscr{G}_{n, k}=\left\{\cap \mathscr{G}: \mathscr{G} \subseteq \mathscr{G}_{n}\right.$ and $\mathscr{G}$ has exactly $k$ elements $\}$. Set $\mathscr{V}_{n}=\bigwedge_{k=1}^{n} \mathscr{G}_{k, n-k+1}, O_{n}=\bigcup \mathscr{V}_{n}$, and $T_{n}=\bigcup_{x \in O_{n}}\left[\{x\} \times\left(\left(\cap\left\{G \in \bigcup_{k=1}^{n} \mathscr{G}_{k}: x \in G\right\}\right) \cap O_{n}\right)\right]$ whenever $n \in \mathbf{N}$. Observe that for each $n \in \mathbf{N}, \mathscr{V}_{n+1}$ is a partial refinement of $\mathscr{V}_{n}$. Fix $x \in X$. Set $n_{x}=\min \left\{k+l: \operatorname{ord}\left(x, \mathscr{G}_{l}\right)=k\right\}$ and let $k_{x}, l_{x} \in \mathbf{N}$ be such that $n_{x}=$ $k_{x}+l_{x}$ and $\operatorname{ord}\left(x, \mathscr{G}_{l_{x}}\right)=k_{x}$. Note that $x$ belongs to exactly one member, say $G$, of $\mathscr{G}_{l_{x}, k_{x}}$. Since $\mathscr{V}_{n_{x}-1}$ is a partial refinement of $\mathscr{G}_{l_{x}, k_{x}}$, we have st $\left(x, \mathscr{V}_{n_{x}-1}\right) \subseteq G$. Next we show that $x \in O_{n_{x}-1}$. Otherwise there is $k \in \mathbf{N}$ such that $k \leq n_{x}-1$ and $x \notin \bigcup \mathscr{G}_{k, n_{x}-k}$. Therefore ord $\left(x, \mathscr{G}_{k}\right)<n_{x}-k$ and $k+\operatorname{ord}\left(x, \mathscr{G}_{k}\right)<n_{x}-$ a contradiction. Thus $x \in O_{n_{x}-1}$ and $x \in T_{n_{x}-1}(x)$. If $x \in T_{n_{x}-1}(y)$ for some $y \in O_{n_{x}-1}$, then there is $H \in \mathscr{V}_{n_{x}-1}$ such that $y \in H$. Consequently $T_{n_{x}-1}(y) \subseteq H$ and $H \subseteq \operatorname{st}\left(x, \mathscr{V}_{n_{x}-1}\right) \subseteq G$. We deduce that $\operatorname{st}\left(x, \mathscr{T}_{n_{x}-1}\right) \subseteq G$. Hence the decreasing sequence $\left(T_{n}\right)_{n \in \mathbf{N}}$ of transitive partial neighbornets on $X$ has all the necessary properties with respect to the cover $\mathscr{C}$ of $X$. We conclude that $X$ is ortho-refinable.

The following proposition generalizes Gruenhage's result that each regular countably compact space with an ortho-base is compact [6, Theorem 3]. Recall that the Lindelöf degree of a topological space $X$, denoted by $L(X)$, is defined as the smallest infinite cardinal $\kappa$ such that every open cover of $X$ has a 
subcollection of cardinality $\leq \kappa$ which covers $X$. A generalization of the Lindelöf degree is the extent $\mathrm{e}(X)$, defined as follows: $\mathrm{e}(X)=\sup \{|D|: D \subseteq X$, $D$ is closed and discrete $\}+\omega$.

Proposition 10. The Lindelöf degree $L(X)$ of an ortho-refinable space $X$ equals its extent $\mathrm{e}(X)$. A countably compact ortho-refinable space is compact.

Proof. It suffices to verify the first statement, because an obvious modification of the given argument proves the second assertion (essentially, replace " $\mathrm{e}(X)$ " by "finite").

Let $\mathscr{C}$ be an open cover of $X$ without subcover of cardinality e $(X)$, and let $\left(T_{\alpha}\right)_{\alpha<\delta}$ be a decreasing chain of transitive partial neighbornets on $X$ witnessing ortho-refinability of $X$ with respect to $\mathscr{C}$. For each $x \in X$ let $\alpha_{x}$ be the minimal $\alpha<\delta$ such that $\operatorname{st}\left(x, \mathscr{T}_{\alpha}\right) \subseteq U$ for some $U \in \mathscr{C}$. For any $\alpha<\delta$ set $F_{\alpha}=\left\{x \in X: \alpha_{x}=\alpha\right\}$. Define inductively for each $\varepsilon<\mathrm{e}(X)^{+}$(the cardinal successor of $\mathrm{e}(X))$ an ordinal $\alpha_{\varepsilon}<\delta$ and a subset $A_{\alpha_{\varepsilon}}$ of $F_{\alpha_{\varepsilon}}$ such that $\left|A_{\alpha_{\varepsilon}}\right| \leq \mathrm{e}(X)$ and $\bigcup_{\beta<\alpha_{\varepsilon}} F_{\beta} \subseteq \bigcup_{s \leq \varepsilon} \operatorname{st}\left(A_{\alpha_{s}}, \mathscr{T}_{\alpha_{s}}\right) \subseteq \bigcup \mathscr{D}_{\varepsilon}$, where $\mathscr{D}_{\varepsilon}$ is a subcollection of cardinality $\leq \mathrm{e}(X)$ of $\mathscr{C}$. Assume that $\alpha_{s}, A_{\alpha_{s}}$, and $\mathscr{D}_{s}$ for all $s<\varepsilon$ are defined. Set $E_{\varepsilon}=X \backslash \bigcup_{s<\varepsilon} s t\left(A_{\alpha_{s}}, \mathscr{T}_{\alpha_{s}}\right)$, and let $\alpha_{\varepsilon}$ be the minimal $\alpha<\delta$ such that $E_{\varepsilon} \cap F_{\alpha} \neq \varnothing$. Suppose that $x \in E_{\varepsilon} \cap \overline{F_{\alpha_{\varepsilon}}}$. By the definition of $\alpha_{\varepsilon}$, we have $\alpha_{x} \geq \alpha_{\varepsilon}$. In fact since $T_{\alpha_{\varepsilon}}(x) \cap F_{\alpha_{\varepsilon}} \neq \varnothing$, it follows that $x \in F_{\alpha_{\varepsilon}}$ from the definition of $F_{\alpha_{\varepsilon}}$. Hence $E_{\varepsilon} \cap F_{\alpha_{\varepsilon}}=E_{\varepsilon} \cap \overline{F_{\alpha_{\varepsilon}}}$ is closed in $X$. By Zorn's Lemma there is a subset $A_{\alpha_{\varepsilon}}$ of $E_{\varepsilon} \cap F_{\alpha_{\varepsilon}}$ such that (1) if $x, y \in A_{\alpha_{\varepsilon}}$ with $x \neq y$, then $x \notin \operatorname{st}\left(y, \mathscr{T}_{\alpha_{\varepsilon}}\right)$ and (2) $E_{\varepsilon} \cap F_{\alpha_{\varepsilon}} \subset \operatorname{st}\left(A_{\alpha_{\varepsilon}}, \mathscr{T}_{\alpha_{\varepsilon}}\right)$. Since $A_{\alpha_{\varepsilon}}$ is closed and discrete in $X,\left|A_{\alpha_{\varepsilon}}\right| \leq \mathrm{e}(X)$. We obtain $\mathscr{D}_{\varepsilon}$ by adding to $\bigcup_{\gamma<\varepsilon} \mathscr{D}_{\gamma}$ a collection of cardinality $\leq \mathrm{e}(X)$ consisting of members of $\mathscr{C}$ that cover $\operatorname{st}\left(A_{\alpha_{\varepsilon}}, T_{\alpha_{\varepsilon}}\right)$. This completes the induction.

Let $\rho$ be minimal $\leq \mathrm{e}(X)^{+}$such that there exists a set $\left\{x_{\varepsilon}: \varepsilon<\rho\right\}$ with an accumulation point $x \in X$, where $x_{\varepsilon} \in A_{\alpha_{\varepsilon}}$ whenever $\varepsilon<\rho$. By the minimality of $\rho$ we have that $\rho$ is a limit ordinal and that $x \in X \backslash \bigcup_{\varepsilon<\rho} \operatorname{st}\left(A_{\alpha_{\varepsilon}}, \mathscr{T}_{\alpha_{\varepsilon}}\right)$. Hence $\alpha_{x}>\alpha_{\varepsilon}$ whenever $\varepsilon<\rho$. Choose $s<\rho$ such that $x_{s} \in T_{\alpha_{x}}(x)$. Then $x \in \operatorname{st}\left(A_{\alpha_{s}}, \mathscr{T}_{\alpha_{s}}\right)$-a contradiction. We conclude that $\mathrm{e}(X)=\mathrm{L}(X)$.

Corollary 5 [13]. The spread of a space having an ortho-base is equal to its hereditary Lindelöf degree.

Remark 1. A slightly modified version of the preceding proof shows that Proposition 10 can be generalized to any topological space $X$ with the property that for each open cover $\mathscr{C}$ of $X$ there is a sequence $\left(\mathscr{U}_{\alpha}\right)_{\alpha<\lambda}$ of open families of $X$ such that $\mathscr{U}_{\beta}$ is a partial refinement of $\mathscr{U}_{\alpha}$ for all $\alpha<\beta<\lambda$, and such that for each $x \in X$ there is an $\alpha<\lambda$ with $x \in \operatorname{st}\left(x, \mathscr{U}_{\alpha}\right) \subseteq C$ for some $C \in \mathscr{C}$. A modification of the proof of Proposition 9 yields that each submetacompact space has this property. Furthermore a modification of the second part of the proof of Proposition 5 proves that each space $X$ with a preortho-pair-base has a sequence $\left(\mathscr{U}_{\alpha}\right)_{\alpha<\lambda}$ of open families of $X$ such that $\mathscr{U}_{\beta}$ is a partial refinement of $\mathscr{U}_{\alpha}$ whenever $\alpha<\beta<\lambda$, and such that for each $x \in X,\left\{\operatorname{st}\left(x, \mathscr{U}_{\alpha}\right): x \in \operatorname{st}\left(x, \mathscr{U}_{\alpha}\right), \alpha<\lambda\right\}$ is a local base at $x$.

In [8] Hart calls a topological space $X$ halvable if for every neighbornet $U$ of $X$ there exists a neighbornet $V$ of $X$ such that for each $x, y \in X$ with $V(x) \cap V(y) \neq \varnothing$ we have that $x \in U(y)$ or $y \in U(x)$. Clearly each monotonically normal space is halvable. 
Proposition 11. Each halvable ortho-refinable space $X$ is paracompact.

Proof. Let $\mathscr{C}$ be an open cover of $X$, and let $\left(T_{\alpha}\right)_{\alpha<\delta}$ be a decreasing chain of transitive partial neighbornets on $X$ witnessing ortho-refinability of $X$ with respect to $\mathscr{C}$. Now set $\alpha_{x}=\min \left\{\alpha<\delta: \operatorname{st}\left(x, \mathscr{T}_{\alpha}\right) \subseteq C\right.$ for some $\left.C \in \mathscr{C}\right\}$ whenever $x \in X$ and define the neighbornet $U=\bigcup_{x \in X}\left(\{x\} \times T_{\alpha_{x} z}(x)\right)$. Let $V \subseteq U$ be an open neighbornet of $X$ that witnesses halvability of $X$ with respect to $U$, and set $\mathscr{D}=\{V(x): x \in X\}$. Moreover fix $y \in X$ and let $\beta=\min \left\{\alpha_{x}: y \in V(x), x \in X\right\}$. Choose $z \in X$ such that $y \in V(z)$ and $\beta=\alpha_{z}$. One readily checks that $\operatorname{st}(y, \mathscr{D}) \subseteq \operatorname{st}\left(z, \mathscr{T}_{\alpha_{z}}\right)$. We conclude that $\{\operatorname{st}(x, \mathscr{D}): x \in X\}$ refines $\mathscr{C}$ and that $X$ is paracompact.

\section{REFERENCES}

1. P. J. Collins, P. M. Gartside, R. D. Kopperman, H. P. A. Künzi, and P. J. Moody, On topologies generated by filters of relations, preprint.

2. S. W. Davis, Spaces with linearly ordered local bases, Topology Proc. 3 (1978), 37-51.

3. P. Fletcher and W. F. Lindgren, Quasi-uniform spaces, Lecture Notes in Pure and Appl. Math., vol. 77, Marcel Dekker, New York, 1982.

4. P. M. Gartside and P. J. Moody, A note on proto-metrisable spaces, preprint.

5. __ Addition to a note on proto-metrisable spaces, preprint.

6. G. Gruenhage, Some results on spaces having an ortho-base or a base of subinfinite rank, Topology Proc. 2 (1977), 151-159.

7. G. Gruenhage and P. Zenor, Proto-metrizable spaces, Houston J. Math. 3 (1977), 47-53.

8. K. P. Hart, More remarks on Souslin properties and tree topologies, Topology Appl. 15 (1983), 151-158.

9. R. W. Heath, D. J. Lutzer, and P. L. Zenor, Monotonically normal spaces, Trans. Amer. Math. Soc. 178 (1973), 481-493.

10. J. Kofner, Transitivity and ortho-bases, Canad. J. Math. 33 (1981), 1439-1447.

11. W. F. Lindgren and P. J. Nyikos, Spaces with bases satisfying certain order and intersection properties, Pacific J. Math. 66 (1976), 455-476.

12. P. J. Nyikos, Some surprising base properties in topology, Studies in Topology (N. M. Stavrakas and K. R. Allen, eds.), Academic Press, New York, 1975, pp. 427-450.

13. M. Sakai, Cardinal functions of spaces with ortho-bases, Tsukuba J. Math. 9 (1985), 167169.

(H. J. K. Junnila) Department of Mathematics, University of Helsinki, Hallituskatu 15, 00100 Helsinki, Finland

E-mail address: HJKJUNNILA@cc.Helsinki.FI

Department of Mathematics, University of Berne, Sidlerstrasse 5, 3012 Berne, SWITZERLAND

E-mail address: KUNZI@MAI. unibe.ch

Institut de Mathématiques, Université de Fribourg, Chemin du Musée 23, 1700 FriBOURG, SUISSE 